\title{
Optimal approach for the interaction between dsos and aggregators to activate der flexibility in the distribution grid
}

\author{
Al-Jassim, Zaid; Christoffersen, Mathias ; Wu, Qiuwei; Huang, Shaojun; Moreno, M a Ángeles; del \\ Rosario, Gerard; Corchero, Cristina
}

\section{Published in:}

Cired - Open Access Proceedings Journal

Link to article, DOI:

10.1049/oap-cired.2017.0307

Publication date:

2017

Document Version

Publisher's PDF, also known as Version of record

Link back to DTU Orbit

Citation (APA):

Al-Jassim, Z., Christoffersen, M., Wu, Q., Huang, S., Moreno, M. A. Á., del Rosario, G., \& Corchero, C. (2017). Optimal approach for the interaction between dsos and aggregators to activate der flexibility in the distribution grid. Cired - Open Access Proceedings Journal, 2017(1), 1912-1916. https://doi.org/10.1049/oapcired.2017.0307

\section{General rights}

Copyright and moral rights for the publications made accessible in the public portal are retained by the authors and/or other copyright owners and it is a condition of accessing publications that users recognise and abide by the legal requirements associated with these rights.

- Users may download and print one copy of any publication from the public portal for the purpose of private study or research.

- You may not further distribute the material or use it for any profit-making activity or commercial gain

- You may freely distribute the URL identifying the publication in the public portal 


\title{
Optimal approach for the interaction between DSOs and aggregators to activate DER flexibility in the distribution grid
}

\author{
$M^{a}$ Ángeles Moreno ${ }^{4}$ \\ ${ }^{1}$ Danish Energy Association, Frederiksberg, Denmark \\ ${ }^{2}$ DTU, Kongens Lyngby, Denmark \\ ${ }^{3}$ IREC, Barcelona, Spain \\ ${ }^{4}$ UC3M, Madrid, Spain \\ $\bowtie$ E-mail: zal@danskenergi.dk
}

Zaid Al-Jassim ${ }^{1} \bowtie$, Mathias Christoffersen ${ }^{1}$, Qiuwei $W u^{2}$, Shaojun Huang ${ }^{2}$, Gerard del Rosario ${ }^{3}$, Cristina Corchero ${ }^{3}$,

\begin{abstract}
The process of predicting the behaviours of distributed energy resources (DER) and controlling them is complex. It will require a huge effort from the distribution system operator (DSO) to establish communication channels to all available DERs in the network and to integrate new ones into the automation system. It is therefore important that a third party (the Aggregator) takes care of the communication with DERs in the network. This study will focus on the following: DSO functionalities that enable communication between the flexibility market and the aggregator; the aggregator role and the functionalities required to be a successful business entity; and the approach (DSO/aggregator model) that was identified and adopted by the IDE4L project. The study concludes and recommends the approach that was adopted by the IDE4L project as the most efficient one to solve congestion and to ensure harmony in the sequence of events. Based on the achieved results, we believe that the IDE4L approach is the optimal method of communication that ensures efficiency, effectiveness, and harmony in communication among the DSO and all other flexibility market players.
\end{abstract}

\section{Introduction}

The number of distributed generators connected to the distribution network has increased at the beginning of this century due to the increased focus on reducing $\mathrm{CO}_{2}$ emissions. Such a rapid increase in generation and demand at the distribution side can cause the distribution system to fail, thus huge investments for network reinforcement become necessary. However, the huge investments can be avoided or postponed if smart grid technologies such as distributed automation systems are adopted. In the IDE4L project, a full-scale automation system have been developed and tested in laboratories as well as in field environments. The developed automation system has a hieratical structure where functions are installed at different voltage levels. However, each level is able to work independently as long as it receives the required input data. At the control centre level, the so-called tertiary controller (TC) is located. The TC manages the operation of the whole medium-voltage (MV) network and is responsible for the communication between the distribution system operator (DSO) and the marketplace.

Fig. 1 illustrates the TC functionalities developed in the IDE4L project.

In this paper, only the market agent (MA) part of the TC will be discussed in detail because it is the only part of the TC that is responsible for the communication between the DSO and market place.

\section{The IDE4L approach}

In the IDE4L project, two approaches have been identified: the aggregator approach and the dynamic tariff (DT) approach. The optimal approach suggested in IDE4L is a combination of those two approaches.

As seen in Fig. 2, there are only two interactions tying the two approaches together. The DSO sends the DT to the retailer part of an aggregator. That means that the aggregator can take the DT into account when offering a service to the market.

The customer is also an actor that can be involved in both the DT approach and the aggregator approach at the same time. This means that the same customer can react to both the DT and to the control signal of the aggregator. In the same manner, the customer can choose to only react to one method or neither. If the customer does not want an aggregator, but chooses to just have a retailer, then the customers can choose to be on a dynamic price, and thus react to the DT, or they can choose to be on a flat rate and not react to the DT. A more complex example could be that the customer has an aggregator. They can choose to offer their flexibility while having a flat rate contract for energy. In this way, the customer would not react on the DT, but only react on specific requests from their aggregator.

\section{Control centre functionalities}

\subsection{Network reconfiguration algorithm}

The network reconfiguration algorithm (NRA) is an important part of the congestion management system at the control centre. However, this paper discusses the interaction between the aggregator and the DSO; therefore, the NRA will not be discussed. Refer to [1] for details about the NRA algorithm and its functionality. 


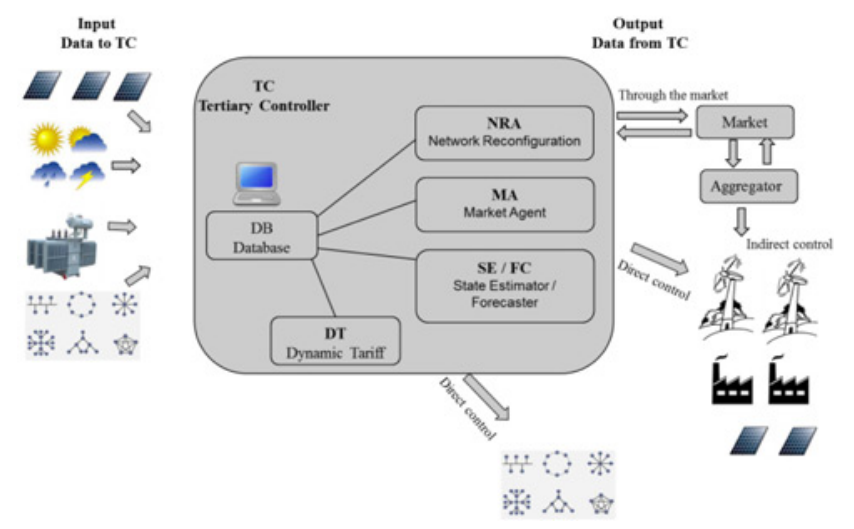

Fig. 1 Control centre functionalities (tertiary controller)

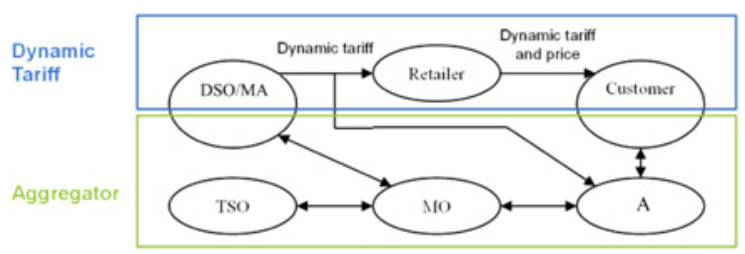

Fig. 2 Approach adopted in the IDE4L project. MO, market operator

\subsection{MA algorithm}

The MA is a function of the TC for MV grid congestion management through the use of DER and demand-side flexibility. The MA algorithm is responsible for the communication between the DSO, the aggregator, and the flexibility market for flexibility purchases. In case the NRA fails to find an optimal solution for a congestion, the MA algorithm communicates with the flexibility market to find the optimal solution, solving an optimal power flow (OPF) problem, which objective function is to minimise the cost of purchased flexibility products. The MA purchases flexibility services like scheduled or conditional re-profiling (CRP) from aggregators in order to influence the power demand, storage, or production within the congested network. Scheduled re-profiling (SRP) products imply an obligation for a specified generation/ demand modification (increase or decrease) at a given time. CRP is the capacity for a specified generation/demand modification (increase and/or decrease) at a given time. The modification is activated by a volume control signal from the buyer.

The MA can work in the day-ahead time frame (offline mode) or in the real-time time frame (online mode).

In the offline mode, the MA can purchase SRPs and CRPs from the flexibility market. The offline MA needs static network data, load and production forecasts, the provisional schedule from the market, and the market clearing prices as inputs. In real-time mode, the MA can activate previously purchased CRPs. State estimation data is used instead of the forecast data as an input [2].

\subsection{Dynamic tariff}

DT is a price signal determined by the DSO to motivate the aggregators of flexible demand to adjust their consumption profile in a way that eliminates congestions [3]. The procedure of using the DT concept to solve the congestion problem in a decentralised manner can be summarised as follows. First, the DSO obtains the flexible demand data, such as energy requirements and the availability, from the aggregators or by its own prediction. The DSO also needs the network information and the predicted spot price. Second, the DTs are calculated through the optimal plan respecting the network constraints, and the DTs are published to all the aggregators. Third, after receiving the DTs, the aggregators make their own optimal plans independently with both the predicted spot prices and the DTs. At last, the aggregators submit their energy plan/bids to the spot market.

\section{Aggregator}

The aggregator is the key mediator between consumers, markets, and other electricity system participants. The aggregator gathers the flexibilities and the contributions provided by prosumers to build active-demand-based products relevant and interesting for flexibility market players such as DSOs. The flexibility of consumers are provided in the form of modifications of their consumption: the aggregator sells a deviation from the forecasted level of demand, and not a specific level of demand.

In order to be able to carry out its duties, the aggregator architecture consists of the following functions:

(i) Consumption forecasting: based on historical consumption data as well as demand models, this tool is used to estimate the baseline consumption used for flexibility services quantification.

(ii) Consumer segmentation: this tool clusters the prosumers portfolio in groups with shared key characteristics.

(iii) Flexibility forecast tool: simulation of the behaviour of the consumers under different price and volume signals.

(iv) Market forecast: forecasting of the market prices.

(v) Optimal planning tool: optimal incentive and bidding policy to maximise the profit of the aggregator.

\subsection{SRP procurement and activation}

SRPs are traded through day-ahead and intra-day markets in the so-called flexibility market. Grid operators (DSOs/TSOs) can access flexibility markets for solving potential congestions arising from day-ahead market results as well as long-term forecast. Aggregators activate these products by means of price incentives sent to their prosumers. The functions and interactions carried out by aggregators during the SRP procurement and activation on day-ahead markets is described below (similar process would apply to intra-day markets with minor changes):

(i) Customer needs/flexibility: aggregators, by means of their flexibility forecasting tool, estimate base load profile, and flexibility availability from their customer portfolio, along with their response to different price incentives. Results from flexibility forecasting will be used for flexibility market bidding processes.

(ii) Flexibility bids: when the flexibility market gate opens, aggregators submit SRP flexibility bids to the flexibility market. These bids can include both flexibility 'up' and flexibility 'down'. From the optimal planning tool, aggregators obtain the available flexibility to be offered to the market together with its price level, which will vary as a function of the amount of flexibility offered. (iii) Send price incentives to $D E R /$ demand: the aggregator enforces accepted flexibility offers by means of sending out the required price incentives among its prosumers portfolio.

\subsection{CRP procurement and activation}

Owing to the low number of prosumers providing flexibility services today, liquidity of local distribution network CRP markets during the coming years is expected to be low. Therefore, in a first stage, the exchange of CRPs is considered to be made by means of bilateral contracts or call for tenders.

There is no specific time where CRP products can be traded or activated. In the CRP procurement, communication between CRP purchasers (DSO) and aggregators is established and contracts are made. Afterwards, in case certain CRP product is activated, two situations are envisioned:

(i) CRP level is below a threshold agreed between the TSO and the DSO: since the activated volume does not have a relevant impact on 
the transmission system, the TSO does not need to technically validate the DSO's CRP purchase. The DSO simply sends a CRP activation request to the aggregators.

(ii) CRP level is above the threshold: the DSO sends a CRP validation request to the TSO before its activation. When TSO real-time validation is finished, it sends one of three signals to the aggregator unit: approved, rejected, or need for curtailment.

\section{Simulations}

\subsection{Network model}

The study case of this work is based on the European MV distribution benchmark [4], reproduced in Fig. 3. Only feeder 1 is considered with voltage limits $\pm 7 \%$. Lines and transformer parameters have been taken from [4]. Transformer is rated $25 \mathrm{MVA}$ and lines 5 MVA. Additionally, the European low-voltage (LV) distribution benchmark has been adopted and adapted to represent the network downstream of node 6 in the MV grid, shown also in Fig. 3. Only the residential subnetwork is considered. The LV network is expanded with two more identical residential subnetworks. Cable and transformer parameters have been taken from [4], except that the transformer off nominal turns ratio is set to $1.0 / 1.03$ to reduce risk of undervoltages. Voltage limits are set to $1 \pm 0.10 \mathrm{p}$.u. Voltage magnitude in the slack node is the same as in node 6 in the MV network. The capacity of the cables from R1 to R9 is 276 and $109 \mathrm{kVA}$ for the rest of cables. The network model and simulation has been implemented in MATPOWER [5].

\subsection{Aggregator and MA results}

The customers of this study grid are divided into two main types: residential $(R)$ or commercial/industrial $(C / I)$. Table 1 shows the demand parameters considered. For each type of load, a certain

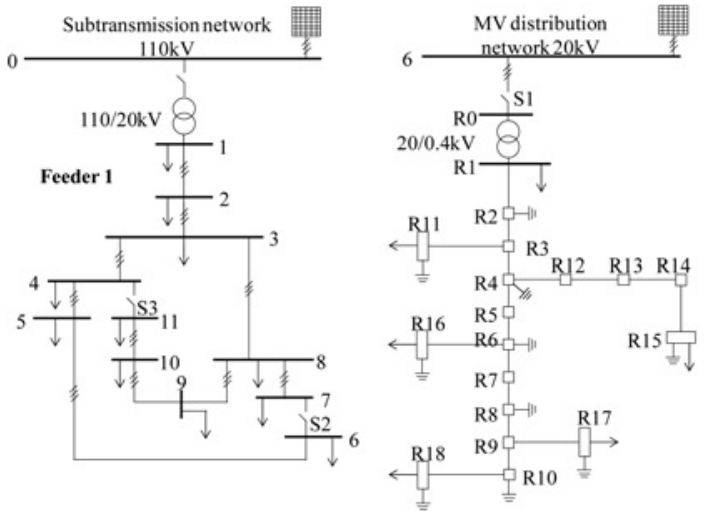

Fig. 3 Topology of the MV distribution network (left) and LV distribution network (right)

Table 1 Demand parameters

\begin{tabular}{|c|c|c|c|c|}
\hline \multirow[t]{2}{*}{ Node } & \multicolumn{2}{|c|}{$\begin{array}{c}\text { Apparent power, } S, \\
\text { kVA }\end{array}$} & \multicolumn{2}{|c|}{ Power factor, pf } \\
\hline & $R$ & $C / I$ & $R$ & $C / I$ \\
\hline 1 & 14,535 & 4845 & 0.98 & 0.95 \\
\hline 2 & - & - & - & - \\
\hline 3 & 399 & 265 & 0.97 & 0.85 \\
\hline 4 & 623 & - & 0.97 & - \\
\hline 5 & 1050 & - & 0.97 & - \\
\hline 6 & 791 & - & 0.97 & - \\
\hline 7 & - & 90 & - & 0.85 \\
\hline 8 & 847 & - & 0.97 & - \\
\hline 9 & - & 675 & - & 0.85 \\
\hline 10 & 686 & 80 & 0.97 & 0.85 \\
\hline 11 & 476 & - & 0.97 & - \\
\hline
\end{tabular}

percentage of flexibility is assumed: $40 \%$ of residential demand and $42 \%$ of commercial/industrial demand. It is also assumed that flexible loads maintain a constant power factor. Different generation units are installed in this MV grid, following [4]. The largest DER unit is a $1500 \mathrm{~kW}$ wind turbine unit, connected in node 7.

Assume that two different aggregators collect the flexibility from each type of customers ( $R$ aggregator and $C / I$ aggregator) and that bilateral contracts have been signed between the DSO and the aggregators. Assume also that the activation of the $C / I$ flexibility has a lower activation price than for $R$ flexibility.

In this case study, it is assumed that an unforeseen event leaves the wind turbine unit out of service, leading to congestions in the transformer and lines L1-2 and L2-3. Furthermore, voltages at nodes 10 and 11 descend below the lower limit. Hence, the MA activates a total of $819.1 \mathrm{~kW}$ of flexibility to relieve congestions, by using all the flexibility available from the $C / I$ aggregator $(396 \mathrm{~kW})$ and $22.4 \%$ of the flexibility available from the $\mathrm{R}$ aggregator $(423.1 \mathrm{~kW}$ of $1892 \mathrm{~kW})$. The solution of the MA is shown in Table 2, with details about the flexibility accepted. Voltage magnitude and branch loading before and after applying the MA algorithm are shown in Figs. 4 and 5, respectively.

All LV customers represented in the LV network are $R$ type in accordance with node 6 at MV level. Also, load data is reduced to $65 \%$ of its original value in order to adapt to node 6 . Demand power factor is kept at 0.95 in all cases. Flexibility capacity is assumed proportional to the demand at each LV node, thus similar customers in the aggregator's portfolio are homogeneously located in the subnetwork. Furthermore, load from customers not belonging to the aggregators' portfolio is assumed evenly distributed among the different demand nodes. The aggregator allocates activated flexibility on a pro rate basis based on the different customers' capacities. As a result of the previous assumptions, load is curtailed proportionally to the demand at each node in this work. Results on the grid impact of the flexibility allocation carried out by the

Table 2 Solution of the market agent

\begin{tabular}{lcc}
\hline Node & $C / /$ flexibility, kW & $R$ flexibility, $\mathrm{kW}$ \\
\hline 3 & 94 & \\
6 & 32 & 109.8 \\
7 & 241 & \\
9 & 29 & 265.9 \\
10 & & 47.4 \\
11 & 396 & 423.1 \\
total & & \\
\hline
\end{tabular}

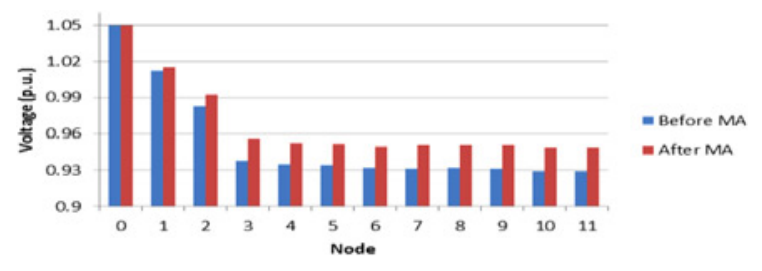

Fig. 4 Voltage magnitude in p.u

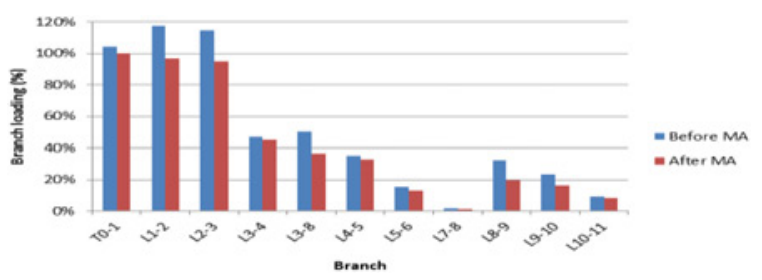

Fig. 5 Branch loading (\%) 
Table 3 Results at LV level ( $R$ aggregator)

\begin{tabular}{lccccc}
\hline & \multicolumn{2}{c}{ Before activation } & & \multicolumn{2}{c}{ After activation } \\
\cline { 2 - 3 } & $\begin{array}{c}\text { Voltages, } \\
\text { u. }\end{array}$ & $\begin{array}{c}\text { Load, } \\
\mathrm{kW}\end{array}$ & & $\begin{array}{c}\text { Voltages, } \\
\text { V. }\end{array}$ & $\begin{array}{c}\text { Load, } \\
\mathrm{kW}\end{array}$ \\
\hline 0 (slack) & 0.932 & - & & 0.950 & - \\
R0 & 0.929 & - & & 0.948 & - \\
R1 & 0.944 & 123.9 & & 0.965 & 106.2 \\
R11 & 0.920 & 9.2 & & 0.945 & 8.0 \\
R15 & 0.872 & 32.4 & & 0.906 & 27.7 \\
R16 & 0.889 & 34.2 & & 0.919 & 29.3 \\
R17 & 0.879 & 21.5 & & 0.912 & 18.4 \\
R18 & 0.874 & 29.3 & & 0.908 & 25.1 \\
total $R$ load (kW) & - & 250.5 & & - & 214.7 \\
$P$ injection R0 (kW) & - & 260 & & - & 220 \\
subnetwork efficiency & - & 96.3 & & - & 97.6 \\
(\%) & - & & & \\
\hline
\end{tabular}

aggregator are shown in Table 3. Only results in one of the three identical subnetworks are shown for simplicity.

Before flexibility activation, there are voltage level violations at MV level (node R0 is below 0.93 p.u.) and LV level (nodes R15R18 are below 0.90 p.u.). Once flexibility is activated, voltage levels are within acceptable levels, with the lowest voltage at node R15 (0.906 p.u.).

Besides, the total residential demand in the subnetwork along with the active power injected to it through node R0 are shown in Table 3. As expected, active power losses have an impact on the power curtailed at MV level, i.e. at the voltage level which triggers the activation due to congestion. In this example, $35.8 \mathrm{~kW}$ are curtailed by the total LV load (from $250.5 \mathrm{~kW}$ down to $214.7 \mathrm{~kW}$ ), but active power injection is reduced by $40 \mathrm{~kW}$ at the MV-LV interconnection (from 260 to $220 \mathrm{~kW}$ ).

\subsection{DT results}

The DT method for day-ahead congestion management is initiated by the DSO. Before the beginning of the day-ahead market, which handles the majority of the energy trades in a deregulated electricity market, the DSO will forecast several basic data which will affect the network operation in the next day. The forecast data includes the availability of flexible demands, such as EV availability shown in Fig. 6. Heat pumps are assumed to be installed in each household in the distribution network. Assume there are two aggregators, where one represents $20 \%$ of the flexible demand, while the other aggregator represents the remaining $80 \%$. The base price, i.e. the day-ahead energy price, is forecasted and shown in Fig. 6. In this case study, the transmission capacity for each line is assumed to be 7 MVA. Without applying the DT method, the energy consumption profiles, including the
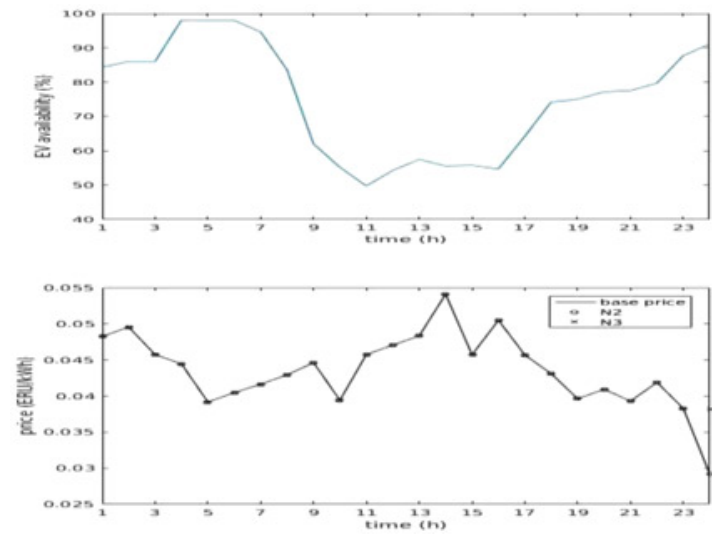

Fig. 6 (Top) EV availability; (bottom) base price, which is forecast day-ahead energy price
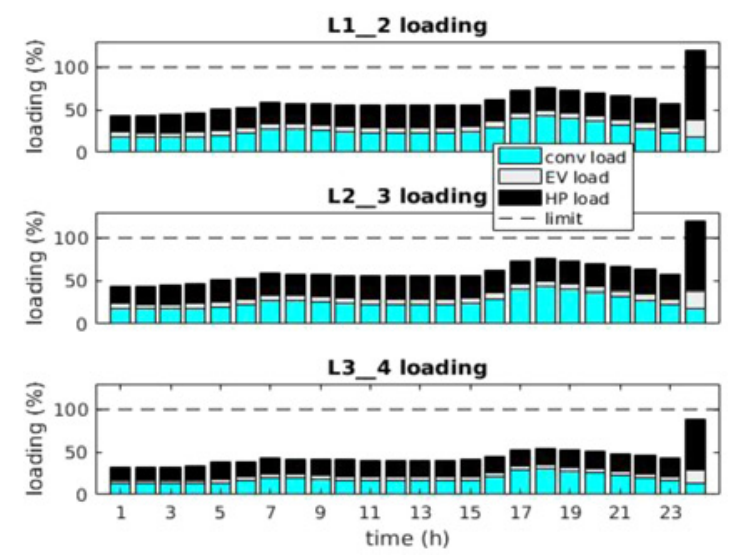

Fig. 7 Forecast energy consumption profile without using the DT method

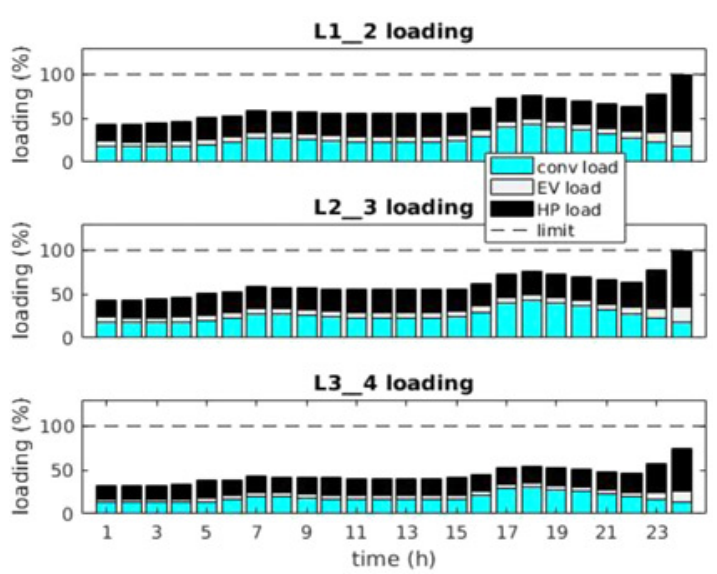

Fig. 8 Forecast energy consumption profile after using the DT method

conventional loads and the flexible demands of aggregators 1 and 2 , are forecasted and shown in Fig. 7 with three selected lines. It can be seen that there are congestions on L1-2 and L2-3 at hour 24; because the forecast energy price at hour 24 is the cheapest and many flexible demands (mainly EVs) chose to consume energy at that hour. Hence, the DSO determines DTs, which raises forecast nodal prices for congested nodes, such as N3 shown in Fig. 6. In this way, the congestions are relieved by the DT method and the results are shown in Fig. 8. As there is no congestion at $\mathrm{N} 2$, the nodal price for $\mathrm{N} 2$ is the same as the base price (Fig. 6).

\section{Discussion}

Specifying the DT in such a way that it operates outside of the market place can create further considerations regarding the method of communication between the DSO and retailers. In this paper, we recommend that communication regarding the DT should not go through the aggregator or be tied to a flexibility market. In this way, all retailers can get access to the new tariff. With the aggregator approach, it is possible that several aggregator bids are aggregated together to deliver one combined service. This will be necessary in areas with a lot of small aggregators, or if the DSO/TSO have a large demand. Also it could be cheaper to activate, e.g. three small bids instead of one large bid. Even though this paper describes a state of art solution, it should not be seen as a final solution. As the amount of DER increases and aggregators form and mature, our knowledge and 
understanding of how to structure a market structure to support them will evolve.

\section{Conclusion}

The benefit of integrating the different time scales (day-ahead, intra-hour, and real-time horizons) according to the operation of aggregators, DSO, TSO, and other third parties lead to minimal adjustments in real time, which are the most demanding. On the other hand, foreseeable occurrences are anticipated and corrected based on historic knowledge and longer term forecasting. The proposed operation merges technical and business knowledges and decisions, while maintaining the interests and competences well separated. The IDE4L approach combines the DT and aggregator approaches. We believe that the DT approach should be seen as a new market condition that in many cases can solve congestion issues for the DSO and is cheap for the DSO to implement. The aggregator approach is a different method that is able to compete with internal technical DSO solutions.

\section{References}

1 Alonso, M.: 'Optimal network reconfiguration for congestion management optimization in active distribution networks'. Int. Conf. on Renewable Energies, May 2016. ISSN 2172-038 X, No. 14

2 IDE4L 2015, deliverable 5.2/3: 'Congestion management in distribution networks'

3 Huang, S.: 'Distribution locational marginal pricing through quadratic programming for congestion management in DN', IEEE Trans. Power Systems, 2015, 30, (4), pp. $2170-2178$

4 CIGRE TF C6.04.02 2013: 'Benchmark systems for network integration of renewable and distributed energy resources', Technical Brochure TB-575, vol. 2, pp. 133-139

5 Zimmerman, R.D., Murillo-Sánchez, C.E., Thomas, R.J. 'MATPOWER: steady-state operations, planning and analysis tools for power systems research and education', IEEE Trans. Power Syst., 2011, 26, (1), pp. 12-19 